\title{
A UTILIZAÇÃO DE AGENTES INTELIGENTES NO APOIO AO AUTOCUIDADO DE IDOSOS
}

Fabiana Santiago Sgobbi, PPGIE, UFRGS, santiagofabiana@hotmail.com

Felipe Becker Nunes, PPGIE, UFRGS, nunesfb@gmail.com

Liane Margarida Rockenbach Tarouco, PPGIE, UFRGS, liane@penta.ufrgs.br

Resumo. Este artigo apresenta um projeto de pesquisa em desenvolvimento que objetiva proporcionar informações e orientações com vistas a promover melhorias no autocuidado do paciente idoso usando um mundo virtual, com a presença de agentes inteligentes. O desenvolvimento, baseado no mundo virtual OpenSim, utiliza entidades programáveis (Non Player Characters) como agentes virtuais para interação com o usuário. O ambiente inclui ferramentas como o "controlador de atividade física", que recebe dados de um dispositivo externo que mede a atividade diária completa do paciente. $\mathrm{O}$ agente virtual, que usa o serviço de um chatterbot, interage com o usuário apresentando dados derivados das medições, bem como orientando sobre autocuidado.

Palavras-Chave: Mundos Virtuais, Autocuidado, Pacientes Idosos.

\section{USING INTELLIGENT AGENTS IN SUPPORT OF SELF-CARE FOR THE ELDERLY}

\begin{abstract}
This paper presents a research project under development that aims to provide information and guidance in order to promote improvements in self-care of elderly patients using a virtual world with the presence of intelligent agents. The development, based on the OpenSim virtual world, uses programmable entities (Non Player Characters) and virtual agents to interact with the user. The environment includes tools like "controller physical activity" that receives data from an external device that measures the complete daily activity of the patient. The virtual agent that uses the service of a chatterbot, interacts with the user presenting data derived from measurements as well as advising on self-care.
\end{abstract}

Keywords: Virtual Worlds, HealthCare, Elderly Patients.

\section{INTRODUÇÃO}

O envelhecimento populacional é um processo que está em andamento em todo o mundo, mas não está ocorrendo da mesma forma em todos os países. O Brasil é um país com uma taxa de envelhecimento considerado moderado avançado. No ano de 2010, o Censo Demográfico contabilizou 14 milhões de pessoas, com 65 anos ou mais, esta faixa representa $7 \%$ da população total. Projeções para as próximas décadas estimam que em 2020, os idosos serão 19 milhões e irão corresponder a $9 \%$ da população total. Já em 2040, estima-se que atingirão 38 milhões, o equivalente a 18\% da população total (IBGE, 2013).

Dentre as soluções existentes que podem auxiliar no que concerne ao auto aprendizado e mecanismo de ajuda aos idosos, estão os mundos virtuais, que oferecem a possibilidade de apresentar uma interface mais amigável e enseja a sensação de acompanhamento ao paciente por agentes de saúde virtuais com comportamento, dicas similares a agentes de saúde humanos (SIRIARAYA, 2014); (POUKE e HÄKKILÄ, 2013). 
O paradigma dos mundos virtuais imersivos já tem sido usado como recurso educacional, em especial na área da saúde e começa também a ser experimentado para uso direto com idosos para fins de apoio e incentivo ao autocuidado da saúde.

A partir da percepção desta realidade foi delineada a pesquisa, ora relatada que investigou as possibilidades técnicas e operacionais de desenvolvimento de um ambiente imersivo (um mundo virtual), voltado ao apoio de pacientes idosos com doenças crônicas. O objetivo da pesquisa foi avaliar o impacto do uso desta tecnologia de mundos virtuais na qualidade de vida (real) desta população alvo e as melhorias proporcionadas em suas condições gerais.

A pesquisa investigou as características desejáveis em um ambiente que se configure como amigável, onde há a disponibilização de orientações e instruções, além de viabilizar a apropriação de informações que permitam acompanhar e monitorar, de forma não intrusiva, algumas doenças crônicas. Para o acompanhamento e monitoração das atividades no âmbito do mundo virtual foi usado o recurso de agentes virtuais, implementados como Non Player Character (NPC), uma possibilidade no mundo virtual, Open Sim, que foi utilizado na pesquisa.

Também foi utilizado um dispositivo sensor de atividade física, "Fit Bit One”, que provê dados de monitoração que foram transferidos para o mundo virtual. Assim, os dados providos pelo equipamento de monitoramento puderam ser disponibilizados no mundo virtual, onde o usuário podia acessá-los e discuti-los com um agente virtual com o objetivo de analisar a situação do paciente e receber aconselhamento, instruções de autocuidado e motivação.

Para ampliar a capacidade de diálogo do agente virtual foi estabelecida uma interconexão do NPC com um chatterbot, cuja base de conhecimento foi preparada para oferecer respostas relevantes ao contexto do experimento realizado, que trata das orientações e instruções de autocuidado, além de viabilizar a apropriação de informações que permitam um monitoramento.

O grupo populacional dos idosos, embora estejam aquém das demais faixas etárias em termos de alfabetização digital, utiliza computador e a Internet de forma crescente a cada ano, conforme indicam pesquisas Em relação ao uso da Internet, dados do Netview (Thiele, 1991) e IBOPE Media (Johnson, 1999), apontam crescimento no número de usuários idosos da rede nos últimos anos. Em janeiro de 2013, eles representaram 1,95\% do total de internautas brasileiros, o que revela uma alta de $8,3 \%$ na comparação com o mesmo mês do ano anterior.

Pensando neste novo perfil de idosos, conforme relatado anteriormente, o sistema tem como meta proporcionar o conhecimento a fim de ensejar as informações e orientações com vistas a promover melhorias no autocuidado do paciente. O ambiente tem ferramentas como o controlador de atividade física, que mede a sua atividade diária completa, incluindo os passos dados, a distância percorrida, as calorias queimadas e os degraus subidos.

Outros dispositivos, tais como balança digital, medidor de pressão também podem enviar informações derivadas de medições por WI-FI ou Bluetooth e estes dados podem então ser disponibilizados no ambiente virtual, (estamos em processo de desenvolvimento, no que tange estes equipamentos). $\mathrm{O}$ mundo virtual tem uma integração com sistemas de acompanhamento (consultas, vacinas etc.) e nutrição (dicas alimentares).

Este artigo está organizado da seguinte maneira: na seção 2 são apresentados alguns trabalhos envolvendo mundos virtuais e a área da saúde; na seção 3 um levantamento teórico dos principais tópicos envolvidos neste projeto é realizado; a 
seção 4 apresenta o método e desenvolvimento dos trabalhos realizados até o momento; por fim, na seção 5 são apresentadas as conclusões deste trabalho.

\section{TRABALHOS CORRELATOS}

Atualmente muito tem se falado sobre a utilização de ambientes imersivos, como facilitadores de aprendizagem. Estudos têm demonstrado que as simulações podem levar ao aumento da autoconfiança, por parte dos pacientes e julgamento clínico melhorado e habilidades para resolver problemas (NETVIEW, 2014) (IBOPE MEDIA, 2014).

Em ambientes imersivos é possível usar diversas ferramentas de apoio, além de palestras, dicas e um monitoramento constante de um "avatar orientador", programado para cuidar dos horários das medicações (por exemplo). Adicionalmente os usuários podem ter a oportunidade de interagir e dialogar com outras pessoas que estiveram conectadas no mundo virtual. Muitas pesquisas já foram feitas em relação a ambientes imersivos e saúde, nas quais os resultados tem sido positivos, como pode ser visto em Schmitz (2014), Rogers (2011) e Machado et al. (2009), sendo um campo onde há ainda bastante espaço a ser explorado (BEARD, 2012).

Pesquisas realizadas por Lukas Roger (2011) indicaram que o uso de simulações de clínicas constitui ferramentas de aprendizagem poderosas por causa de sua capacidade de expandir a experiência do acadêmico da saúde. As simulações clínicas fornecem uma aprendizagem baseada na prática.

Além dos benefícios dos tradicionais simuladores de clínicas, a simulação é uma solução flexível e financeiramente acessível, visto que se trata de um ambiente cooperativo e colaborativo de aprendizagem. Ao contrário das tecnologias tradicionais, sistemas imersivos de multiusuários de ambientes virtuais podem ensejar uma experiência mais realista do ambiente no qual se busca capacitar os usuários. No caso da experiência realizada por Beard (2012), relata um conjunto de experiências utilizado o mundo virtual Second Life, para incorporar materiais de aprendizagem com estratégias eficazes, permitindo que estudantes da área da saúde obtenham uma experiência simulada de clínica, em um ambiente de imersão virtual.

Na última década, os ambientes imersivos baseados em simuladores se tornaram uma plataforma popular para recriar uma experiência clínica em educação na saúde, pois têm um custo relativamente baixo, permitem a aprendizagem flexível, têm uma abordagem centrada no aluno e promovem o engajamento na aprendizagem ativa (BENSON, 2004).

O projeto relatado, neste artigo aborda o uso de mundos virtuais no escopo da saúde, apresentando como elemento diferenciador o uso de agentes virtuais implementados como Non Player Characters no ambiente Open Sim integrados com um chatterbot com uma base de conhecimento personalizada possibilitando que o usuário possa aprender com o agente a realizar os autocuidados. Adicionalmente foi usado neste experimento o dispositivo "Fit Bit One” que provê informações sobre o nível de atividade do usuário humano e estas informações estão sendo integradas ao ambiente do mundo virtual podem ser acessadas pelo próprio usuário e pelo agente virtual não somente para acompanhar e comentar as atividades realizadas, mas também para incentivar e sugerir pequenas sequências de exercícios funcionais que complementem as necessidades de atividade do usuário-paciente. 


\section{FUNDAMENTAÇÃO TEÓRICA}

Com a manipulação de réplicas do mundo real, os pacientes e profissionais de saúde, podem tomar conhecimento e experimentar situações perigosas com segurança e monitoração. Pesquisas recentes de Paas e Sweller (2011) mostraram que atividades como a comunicação, o reconhecimento de movimentos humanos e o uso de gestos, decorrentes de motivações intrínsecas humanas, constituem atividades primárias e requerem menor esforço cognitivo para o seu processamento na memória de trabalho.

Por outro lado, as atividades primárias auxiliam no processamento das informações em atividades secundárias, que são mais complexas e decorrem de uma aprendizagem estruturada e que necessitam de motivações externas para gerar um efetivo envolvimento dos estudantes.

A possibilidade de observar avatares em um ambiente imersivo e de aprender a partir desta observação oferece uma alternativa importante para a aquisição de conhecimentos na área de saúde com vistas a criar melhores condições para que os pacientes se tornem capazes de autocuidado. O ser humano utiliza a comunicação como um meio para apropriar-se e difundir conhecimentos, fato que foi discutido na teoria sócio-histórica de Vygotsky, conforme discutido por Baquero (1998).

Segundo Vygotsky, todas as atividades cognitivas básicas do indivíduo ocorrem de acordo com sua história social e acabam se constituindo no produto do desenvolvimento histórico-social de sua comunidade (BAQUERO, 1998). Paas e Sweller (2011) acrescentam que a comunicação funciona como base para o envolvimento em atividades colaborativas, com as quais os indivíduos unem as forças de sua memória de trabalho e tornam-se aptos a resolver problemas de maior complexidade.

Além da comunicação, uma atividade primária de suma importância para a promoção da aprendizagem é a imitação, sendo esta considerada a chave para a evolução humana na concepção de Tomasello e Haberl (2003). Paas e Sweller (2011) afirmam que, ao assistir a realização de tarefas por outro indivíduo o sujeito torna-se capaz de processar os passos realizados, reorganizando-os em esquemas a serem armazenados em sua memória de longo alcance. Salientam que é necessária uma base de conhecimento relacionada à tarefa em questão para que esta aptidão torne-se viável.

Estes pressupostos teóricos serviram de base para o desenho do ambiente projetado e construído usando o metaverso OpenSim. Os metaversos, também conhecidos como ambientes imersivos oferecem ao usuário espaços tridimensionais, nos quais se pode transitar, interagir e vivenciar experiências em um mundo que só existe no contexto virtual. No mundo virtual em um ambiente imersivo, pacientes podem assistir vídeos, interagir com instrutores (através de seu avatar), para juntos discutirem e engajarem-se na resolução de problemas. Eles interagem, no metaverso através de seus avatares, o que proporciona a sensação de compartilharem um ambiente com atividades colaborativas (TAROUCO et al., 2012).

Em mundos virtuais, o indivíduo pode observar a realização de tarefas efetuadas por outros avatares e assim aprender com as mesmas. Além disso, pode ter acesso a demonstrações criadas a partir de animações disponibilizadas no ambiente, acompanhar e monitorar os medicamentos que devem ser tomados, receber dicas de profissionais da saúde, entre tantos outros recursos possíveis. Os processos possíveis de monitoração irão envolver também outros dispositivos capazes de medir e transmitir sinais diretamente (balanças, medidores de pressão, controladores de atividades diárias, etc.) seguindo uma tendência atual de conexão de dispositivos na rede e que é denominada Internet das Coisas (TAROUCO et al., 2012). 


\section{MÓDULOS DESENVOLVIDOS E INTEGRAÇÕES REALIZADAS}

Esta pesquisa objetivou investigar condições de aprendizagem de pacientes, em ambientes imersivos, buscando delinear uma estratégia para promover o autocuidado, de pacientes idosos com doenças crônicas.

Para tanto, a primeira etapa deste projeto caracterizou-se pelo levantamento do aporte teórico relacionado às principais questões englobadas neste trabalho, como mundos virtuais, cuidados com idosos e trabalhos relacionados envolvendo estas duas áreas de pesquisa. Após a realização desta etapa, a seleção das tecnologias a serem utilizadas foi executada para dar início ao desenvolvimento do trabalho.

Este projeto foi implementado, em um ambiente virtual baseado em software livre, utilizando o software Open Sim, o qual tem sido utilizado pelo grupo de pesquisa como opção de metaverso por ser atender a necessidades funcionais como: suporte à programação em várias linguagens, comunicação interativa, modelagem gráfica de objetos e inserção de imagens e áudio (TAROUCO et al., 2012).

Adicionalmente, este ambiente enseja o uso de Non Players Characters que permite a inclusão de agentes virtuais para auxiliar nas atividades desenvolvidas no mundo virtual, sendo que os mesmos foram enriquecidos com uma base de conhecimento personalizada para tratar as questões referentes ao escopo deste trabalho. Isto foi conseguido integrando o agente virtual com um chatterbot fornecido pela Pandorabot (2014).

As motivações que levaram a escolha do Pandorabots como suporte para o chatterbot foram, a facilidade de criar "pessoas virtuais", por se tratar de um software livre e de código aberto, além da facilidade de acessá-lo via web, bem como os recursos oferecidos para criar, modelar e publicar chatterbot específicos e disponibilizá-los via Internet. O registro neste ambiente é gratuito e após esta etapa é possível iniciar a criação de seus próprios robôs virtuais (chatterbots). Neste ambiente a base de conhecimento utilizada pelo chatterbot é expressa mediante o uso da linguagem de marcação Inteligência Artificial Markup Language (AIML) que é baseada em XML. Isto facilita a tarefa de personalizar ou criar a partir do zero um chatterbot com conhecimento especializado em determinada área.

Para a conexão do Pandorabots com o Open Sim, primeiramente foi necessária a criação e configuração de uma conta no "Pandorabots", no site: http:/pandorabots.com.

A segunda parte envolveu a criação de um NPC, ou seja, um personagem tal como um avatar, mas que é programável e não manipulável diretamente pelo usuário como ocorre quando o mesmo atua no mundo virtual através de seu avatar. Desta forma, o usuário tem a sensação que esta interagindo com outro avatar, que proporciona um serviço equivalente a uma tutoria constante.

Para conseguir a criação dos NPCs no Open Sim, usando funções que permitam sua animação por scripts foi necessário fazer algumas configurações especiais para realizar a ativação dos NPC. Isto foi realizado mediante edições no arquivo “OpenSim.ini”. As configurações a serem feitas são as seguintes:

- $\quad$ Enabled = true na seção [NPC] .

- $\quad$ Enabled = true na seção [XEngine].

- AllowOSFunctions = true na seção [XEngine]

- $\quad$ OSFunctionThreatLevel = VeryHigh na seção [XEngine]

Para que o avatar possa ser criado no mundo virtual, realize movimentos previamente estabelecidos, emita falas no chat e execute determinadas animações, a 
alteração das seções XEngine e GridInfo neste mesmo arquivo "OpenSim.ini” também se faz necessário, como pode ser visto a seguir:

- Allow_osNpcCreate $=$ true

- Allow_osNpcMoveTo = true

- Allow_osNpcRemove = true

- Allow_osNpcSay = true

- Allow_osAvatarPlayAnimation = true

- Allow_osAvatarStopAnimation = true

Feito isso o arquivo "OpenSim.ini" pode ser salvo. Cabe destacar que tais alterações afetarão todas as regiões. A seguir devem ser editados os "scripts” em um prim objeto do mundo virtual) disparador do NPC. Quando este objeto for tocado pelo avatar será instanciado um NPC.

A programação do NPC (Tabela 1) para a comunicação com o chatterbot do Pandorabot envolve o uso de comandos, como pode ser visto na tabela abaixo, em que o script llGetOwner é capaz de retornar o numero do UUID (numero identificador do avatar). O Id, em questão é gerado no momento da criação do robô, pelo Pandora e o script llListen fica esperando ouvir algum comando para ativá-lo. No momento seguinte, deverá ser adicionado o código que estabeleça o link com o Banco de Dados do Pandorabots (Tabela 2). Este mesmo tipo de conexão ao banco de dados, também foi utilizado para obtenção de dados do equipamento "Fit Bit One".

Tabela 1 - Programação NPC com chatterbot.

Tabela 2 - Conexão banco de dados Pandorabots.
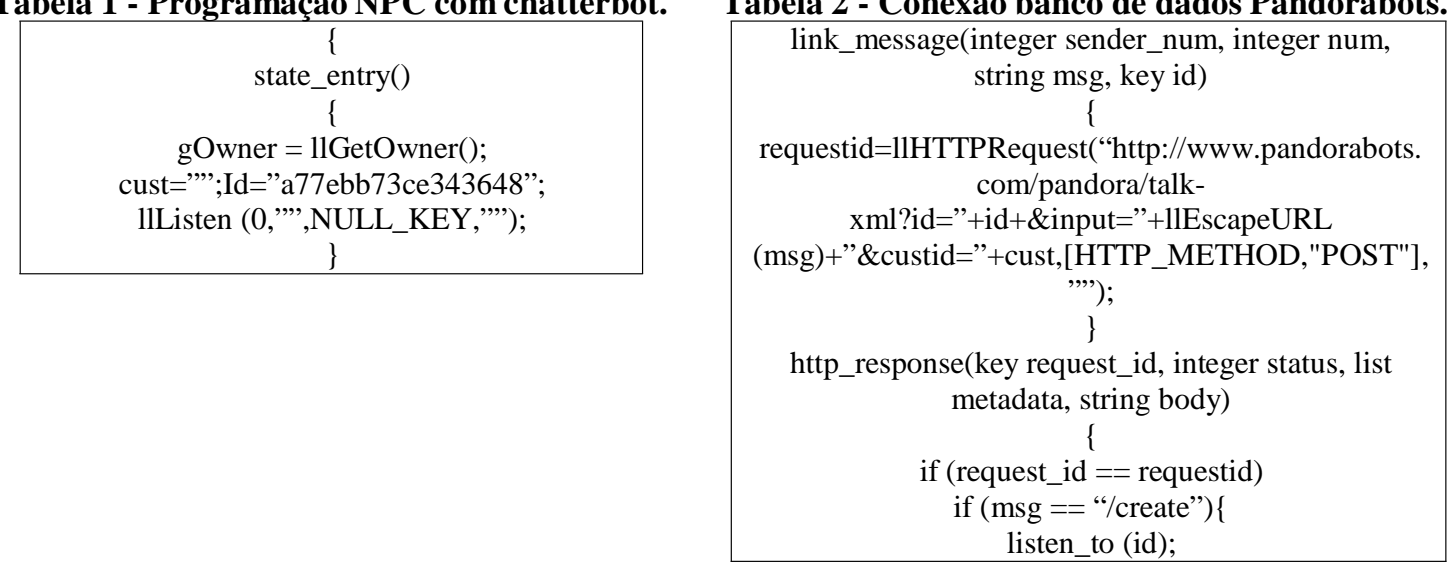

O comando llHTTPRequest executa uma rotina comparativa entre um "termo" localizado na base dados AIML, personalizada pelo programador no site Pandora, usando a pergunta formulada pelo usuário no mundo virtual. Com base nesta comparação realizada, é fornecida para o usuário uma resposta relacionada aos termos relevantes presentes na pergunta.

Na sequência são demonstrados os scripts responsáveis pela criação de um NPC (Tabela 3), através do número identificador do avatar (UUID) para criar um “clone” do avatar paciente. É possível visualizar que no comando osNpcCreate foi atribuído um nome para ele, a coordenada onde deverá ser criado, o seu UUID e uma fala que o npc deverá dizer no momento da sua criação.

A movimentação de um NPC (vide códigos na Tabela 4) pode ser feita de duas maneiras: A primeira envolve a criação e inserção da animação que o NPC exibirá como comportamento. A criação da animação pode ser realizada, com auxílio de software para renderização de movimentos (Ex.: Poser), e, posteriormente adicionado no inventario do ambiente. 
Tabela 3 - Script para criação do NPC.

Npc = osNpcCreate (“Test”, “NPC”, <128,

128, 24>, "b95bf240-e386-4f1d-bc57e5b313ff3d58");

osNpcSay (npc, “Eu estou criado”); return;
Tabela 4 - Movimentos NPC.

osAvatarPlayAnimation (npc, “avatar_backflip”);

llSleep(2); osAvatarStopAnimation (npc, “avatar_backflip”); llSleep(1);

osAvatarPlayAnimation(npc,“bouncy_ball_walk”); llSleep(9);

osAvatarStopAnimation(npc,"bouncy_ball_walk”) ; osNpcSay (npc, "Ending animation sequence...”);

A segunda forma de criar um NPC baseado apenas em "scripts", envolvendo a utilização de uma função pré-estabelecida chamada "osAvatarPlayAnimation”, que também pode chamar uma animação criada, porém oferece a possibilidade de utilização algumas animações previamente estabelecidas.

Com tais codificações, é possível instanciar um NPC, ligado à base de dados do Pandorabots e à base de dados do equipamento "Fit Bit One". A personalização da base de dados AIML do chatterbot está sendo realizada, de forma a intervir nos dados recebidos pelo "FitBit One”. O uso de dispositivos de monitoração de atividade física visou proporcionar condições para monitorar a atividade física, registrando a atividade diária dos usuários.

O software Poser foi utilizado para criar uma renderização 3D para animação e renderização de figuras humanas. Semelhante a um estúdio de fotografia virtual, Poser permitiu que fossem criadas rotinas de atividades físicas, a fim de motivar os idosos para imitar os movimentos.

Com os processos delineados, o desenvolvimento do ambiente teve início e alguns testes preliminares puderam ser realizados para avaliar o andamento do projeto.

A alteração da base de conhecimento do chatterbot foi realizada com a edição de um arquivo construído com a linguagem de marcação AIML. As principais tags do AIML serão listadas abaixo (Tabela 5):

Tabela 5 - Movimentos do NPC.

<aiml> inicia e termina um bloco programado em AIML
<category > identifica uma “unidade de conhecimento" na base de conhecimento
<pattern> identifica um padrão de mensagem simples frequentemente utilizado por usuários
$<$ template > contém a resposta para uma mensagem do usuário

O "controlador de atividade física", utilizado foi o "Fit Bit One": ele mede a atividade diária completa, incluindo os passos dados, a distância percorrida, as calorias queimadas e os degraus subidos. Basta colocá-lo no bolso, na roupa ou no cinto, utilizando o clipe de silicone para fixação. Este equipamento, que pode ser visualizado na Figura 1, também pode registrar o tempo e a forma como o seu usuário dorme, com ferramentas online que ilustram os seus padrões de sono profundo e em vigia.

Na Figura 2, é possível visualizar o avatar interagindo com o "NPC" (avatar tutor), onde está sendo a utilização de AIML, personalizada, onde foi definida mais de uma resposta para um padrão reconhecido na pergunta. 


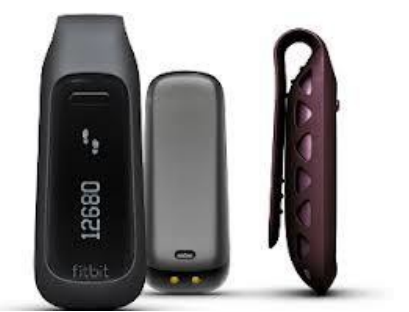

Figura 1 - Equipamento FITBIT

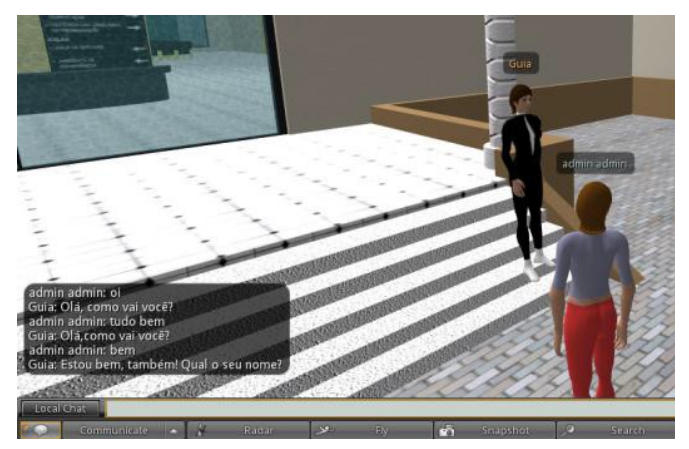

Figura 2 - O NPC interagindo com o paciente

A Figura 3 apresenta a imagem do avatar de um usuário do ambiente em sua casa (no metaverso), acessando o painel de informações de suas atividades. Um NPC, também acessa a cada 5 horas os dados do FitBit One e em caso de grande ociosidade, é disparado uma rotina (gerado pelo software Poser) para estimular o paciente a fazer uma sequência de atividades físicas. Desta forma o paciente terá seus dados monitorados por um NPC, que poderá incentivá-lo a maior atividade, se for o caso.

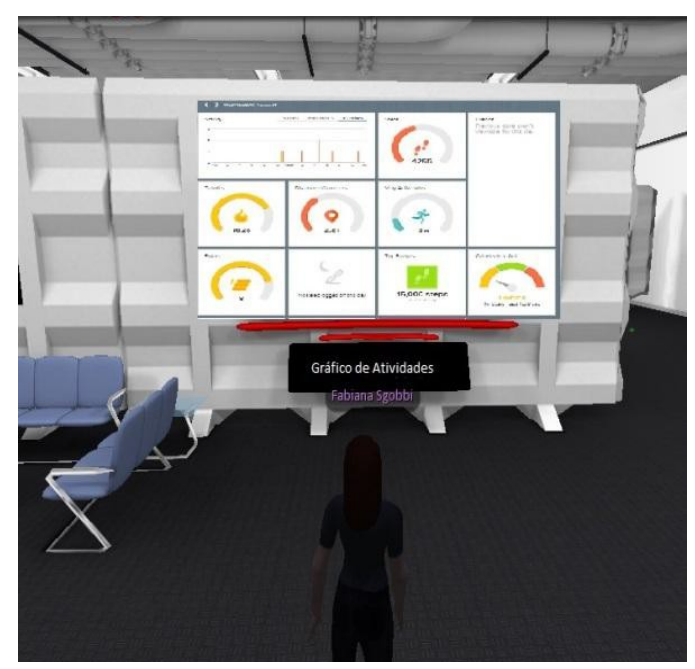

Figura 3 - Avatar acessando seus dados

No que tange à conexão dos dados do equipamento ao mundo virtual, foi realizada com os seguintes procedimentos:

- Configuração de uma planilha do Google e Scripts, visto que este equipamento possui uma API de conexão;

- Configuração de uma conta FitBit desenvolvedor e registrar do aplicativo;

- Cópia das chaves de API (estas serão utilizadas no script do metaverso);

- Autorização do Fit Bit, para o download do Script, da API;

- Fazer download dos dados Fit Bit para uma planilha de formato CSV, com a utilização do PHP, na qual é realizada uma gravação em Banco de dados MYSQL;

- Construção de uma página em "PHP” para consulta ao banco de dados;

- Configuração de um gatilho automático (uma sequencia de comando) para atualizar automaticamente a planilha com novos dados em um horário específico. 
A programação responsável por buscar os dados de um Banco de Dados e levalos ao Open Sim deve ficar em um objeto no mundo virtual, que pode ser disparada periodicamente ou por demanda (requisição do avatar).

O projeto avançou até a implementação de um protótipo que foi avaliado qualitativamente por um pequeno número de pacientes idosos que se sentiram motivados a usar o ambiente, mas não existem ainda dados quantitativos desta avaliação.

\section{CONCLUSÃO}

A evolução ocorrida nas últimas décadas com relação aos mundos virtuais tem permitido o uso dos seus recursos para diferentes fins, como a assistência para idosos com doenças crônicas. Um exemplo disso é a criação de NPCs neste ambiente, que possibilitam a interação destes com os avatares controlados pelos usuários, de forma individualizada ou conjunta, promovendo informações e orientações para promover a autoajuda do usuário.

Com a integração das tecnologias ora apresentadas pretendeu-se ensejar a oferta de um serviço de apoio e incentivo ao autocuidado por idosos. O uso do mundo virtual tem como meta oferecer uma plataforma cativante para que os usuários se sintam motivados a frequentar o ambiente e interagir com o agente virtual alcançando um melhor autoconhecimento e melhorando sua qualidade de vida.

$\mathrm{O}$ artigo apresentou um relato de pesquisa acerca das possibilidades de desenvolvimento de um ambiente imersivo, voltado ao apoio de pacientes idosos com doenças crônicas ou com restrição de movimentação, avaliando o seu impacto na qualidade de vida (real) desta população alvo e as melhorias proporcionadas em suas condições gerais. Para tanto, foi realizada a criação de NPCs no mundo virtual, detalhando todo o seu processo de desenvolvimento e a sua interconexão com uma base de dados AIML personalizada no Pandorabots e com o equipamento de monitoramento FitBit.

Os resultados preliminares demonstraram a viabilidade da proposta de pesquisa, visto que a integração do equipamento FitBit com o mundo virtual foi bem sucedida. Além disso, a criação dos NPCs interligados a base de dados AIML do Pandora bots também foi efetivada, o que permite a realização de testes com usuários reais.

A pesquisa está em andamento e trabalhos futuros, envolverão o prosseguimento da pesquisa com teste e avaliação do protótipo pela faixa de usuário que constitui o público alvo deste projeto.

\section{REFERÊNCIAS BIBLIOGRÁFICAS}

BAQUERO, R. “Vygotsky e a aprendizagem escolar". Porto Alegre (RS): Artes Médicas, 1998.

BEARD, L.; WILSON, K.; MORRA, D.; KEELAN, J. “A Survey of Health-Related Activities on Second Life”. Disponível em: <http://www.jmir.org/2009/2/e17/> Acesso em: 26 de dezembro de 2012.

BENSON, P. “Online learning: a means to enhance professional development". Critical Care Nurse. p 24, 1, 60-63, 2004.

IBGE. “Sobre a condição de saúde dos idosos: indicadores selecionados”. 2010. Disponível em: www.ibge.gov.br/home/estatistica/sociosaude/2009/com_sobre.pdf. Acesso em: 10 de janeiro de 2013. 
JOHSON, J.; ZERWIC, J.; THEIS, S. “Clinical simulation laboratory and adjunct to clinical teaching”. Nurse Educator, p. 24, 5, 37-41, 1999.

MACHADO, L. S.; MORAES, R. M.; NUNES, F. "Serious Games para Saúde e Treinamento Imersivo”. Book Chapter, 2009.

PAAS, F.; SWELLER, J. “An Evolutionary Upgrade of Cognitive Load Theory: Using the Human Motor System and Collaborationt: o Support the Learning of Complex Cognitive Tasks”. Educ Psychol Rev. New Jersey, p. 1-19, 2011.

POUKE, M., HÄKKILÄ, J. "Elderly healthcare monitoring using an avatar-based 3D virtual environment". International Journal of Environmental Research and Public Health, 10 (12), pp. 7283-7298, 2013.

ROGERS, L. "Developing simulations in multi-user virtual environments to enhance healthcare education”. University of Ballarat, P.O. Box 663, Ballarat, Vic. 3353, Australia, v. 42, n. 4, p. 608-615, 2011.

SCHMITZ, Q. T.; KEMCZINSKI, A.; HOUNSELL, M. S. "Realidade Virtual no Treinamento da Inspeção de Focos de Dengue”. IV Workshop de Informática aplicada à Saúde CBComp, Itajaí - SC, Outubro. IV WIS-CBCOMP, v. 1. p. 541-546, 2004.

SIRIARAYA, P., ANG, C. S., BOBROWICZ, A. "Exploring the potential of virtual worlds in engaging older people and supporting healthy aging”. Behaviour and Information Technology, 33 (3), pp. 282-293, 2014.

Site oficial do IBOPE MEDIA. Disponível em: http://www.ibope.com.br/ptbr/ibope/quemsomos/unidadesnegocio/ibopemedia/paginas/ibope-media.aspx. Acesso em: 15 de agosto de 2014.

Site oficial do NetView. Disponível em: http://www.ibope.com.br/ptbr/solucoes/consumodosmeios/internet/paginas/netview.aspx. Acesso em 15 de agosto de 2014.

Site oficial do Pandora Bots. Disponível em: http:/pandorabots.com. Acesso em 14 de junho de 2014.

TAROUCO L. M. R.; ÁVILA, B. G.; AMARAL, E. M. H.; ZEDNIK, H. “VEGA Implementando um Laboratório Virtual Imersivo no OpenSim”. Renote, v. 10., n. $1,2012$.

TAROUCO, L. M. R.; BERTHOLDO, L.; GRANVILLE, L. Z.; ARBIZA, L.; CARBONE, F., MAROTTA, M. "Interoperatibility and Security issues Regarding Internet of Things on Helthcare”. In: 1st IEEE Intenational Workshop on Mobile Consumer Health Care Networks, Systems and Services (MobiCHeSSL2), 2012.

THIELE, J.; HOLLOWAY, J.; MURPHY, D.; PARDAVIS, J; STUCKEY, M. "Perceived and actual decision making by novice baccalaureate students". Western Journal of Nursing Research, v. 13, n. 5, pp. 616-626, 1991.

TOMASELLO, M., e HABERL, K. "Understanding attention: 12- and 18-month olds know what is new for other persons”. Developmental Psychology, 39 (5), 906912, 2003. 